\title{
European Perspectives for Electron-Nucleon Scattering at the Luminosity Frontier
}

\author{
Wolf-Dieter Nowak ${ }^{\mathrm{a} *}$ \\ aDESY Zeuthen, Platanenallee 6, D-15738 Zeuthen, Germany \\ European perspectives are discussed on fixed-target electron or positron scattering experiments using polar- \\ ized and unpolarized beams and targets in various combinations. The goal envisioned is a deep and complete \\ understanding of the momentum and spin structure of hadrons in the context of Quantum Chromo Dynamics, \\ based on measurements at moderate and low photon virtualities. This program can be realized by performing \\ electron-nucleon experiments with high resolution and high luminosity, at beam energies of at least 25-30 GeV.
}

\section{INTRODUCTION}

Over more than two decades the momentum and spin structure of single partons in the nucleon has been investigated by now, preferentially using charged leptons as probe(s). A great variety of measurements, performed in fixed-target and collider experiments, turned Quantum Chromo Dynamics (QCD) from a candidate theory into the widely accepted field theory of strong interactions. Large enough photon virtualities $Q^{2}$ were required to successfully test the abilities of perturbative QCD to describe short-range phenomena. In contrast, the description of long-range phenomena and especially of parton correlations in hadrons, over a broad range in $Q^{2}$, is still in its infancy. It may be expected that contemporary theoretical developments will at some moment evolve into a calculable field theoretical description of hadronic structures. It is hence vital to maintain in this process a permanent confrontation of theoretical ideas to measurements.

In this paper possible ways are discussed how to acquire in the future adequately precise experimental data to test different approaches and to eventually be able to select the correct method describing hadronic structure on the basis of fundamental interactions.

\footnotetext{
*Invited talk at the Workshop 'The Spin Structure of the Proton and Polarized Collider Physics', Trento/Italy, July 23-28, 2001
}

\section{PHYSICS MOTIVATION}

Deep inelastic lepton-nucleon scattering (DIS) continues to be a very successful tool to explore perturbative QCD. On the one hand, a wealth of inclusive data from 'unpolarized' fixed-target and collider experiments at CERN, DESY and FNAL allowed a rather accurate determination of the quark distributions $q\left(x, Q^{2}\right)$ through precise QCD fits of the unpolarized nucleon structure function $F_{2}\left(x, Q^{2}\right)$. Here $x$ is the Bjorken scaling variable describing the constituent's momentum fraction in a fast moving hadron. On the other hand, results from several 'polarized' fixed-target experiments at CERN, DESY and SLAC measuring double spin asymmetries in inclusive DIS cross sections led to the determination of the polarized structure function $g_{1}\left(x, Q^{2}\right)$ with modest accuracy. Although this data is limited to moderate values in $Q^{2}$, it constrains the contribution of the quark spin to the nucleon's helicity on the $10-20 \%$ level of accuracy.

Recent and near-future measurements at CERN (COMPASS), DESY (HERMES) and JLab are changing the emphasis from inclusive DIS to semi-inclusive (SIDIS) measurements. The main thrust of COMPASS will be a determination of the polarized gluon distribution $\Delta G(x)$ [1], over a limited range of $x$ with about $10 \%$ accuracy (cf. Fig. 33). Helicity distributions $\Delta q(x)$ will be available from HERMES, on the few \% level for valence quarks and for the first time for sea 
quarks [2]. A first measurement of the transversity $\delta q(x)$ 3 5 , characterizing the distribution of the quark's spin in a transversely polarized nucleon, is the main goal of HERMES in 2002-03 [6]. Accomplishing a complete understanding of the nucleon structure on the twist-2 level requires precise experimental data for all parton distribution functions mentioned above.

A few years ago Generalized Parton Distributions (GPDs) [7] 10], a set of non-perturbative distribution functions, were introduced in the description of several processes ranging from inclusive to hard exclusive scattering. GPDs embody ordinary parton distribution functions and nucleon form factors. They depend on $Q^{2}$, two longitudinal momentum fractions and $t$, the momentum transfer at the nucleon vertex. GPDs can in principle be revealed from a set of different cross sections and asymmetries for various exclusive processes.

The recent strong interest in GPDs was stimulated by the finding [9] that the total angular momentum carried by partons of a certain type is given by the second moment of the sum of the unpolarized GPDs describing this type of partons, in the limit of small $t$. The total angular momenta carried by quarks and gluons in the nucleon, $J_{q}$ and $J_{g}$, constitute the hitherto missing pieces in the puzzle representing the momentum and spin structure of the nucleon.

Nowadays hard exclusive lepton-nucleon processes appear as a viable tool to access quark GPDs by measuring a photon or a scalar, pseudoscalar or vector meson in the final state. Very recently, first experimental results on Deeply Virtual Compton Scattering (DVCS) have been obtained. The fixed-target data from HERMES 11] and CLAS (JLab) [12] exhibit significant asymmetries in the distribution in the azimuthal angle between lepton and hadron planes, thereby confirming the expected interference between the dominating DVCS handbag diagram and the background Bethe-Heitler process. H1 measured the DVCS cross section at the HERA collider 13] and found agreement with QCD-based calculations [14].

Even after completion of the present series of lepton-nucleon SIDIS measurements 2 both helicity and transversity quark distributions as well as the polarized gluon distribution will remain clearly inferior in accuracy compared to the unpolarized distributions. Concerning GPDs it is generally agreed that because of their complexity every attempt to really measure them will require a huge common effort of theory and experiment.

\section{EXPERIMENTAL FACILITIES}

The above envisioned 'complete' determination of the momentum and spin structure of the nucleon (and other hadrons) clearly calls for new experimental facilities which, in comparison to existing experiments, include

- an accelerator with a high duty factor of at least 5-10\% allowing for hitherto unprecedented luminosities of $10^{35} \mathrm{~cm}^{-2} \mathrm{~s}^{-1}$ or more;

- beams of electrons and, if possible, positrons 3 with an energy definition better than $\mathcal{O}\left(10^{-4}\right)$ and a high degree of polarization;

- beam energies above $25-30 \mathrm{GeV}$ to reach the perturbative regime of a few $\mathrm{GeV}^{2}$ in $Q^{2}$, over the full kinematically accessible $x$ range;

- radiation-tolerant (cryogenic) solid-state targets that can achieve high degrees of longitudinal and transverse polarization;

- a spectrometer that combines very fast, $\mathcal{O}(1-2$ ns) triggering with high-precision tracking and full particle identification.

These requirements are the basis for two proposals, TESLA-N and ELFE@DESY, described in

\footnotetext{
${ }^{2}$ The RHIC $p p$ spin program 15 will provide precise and complementary data on $\Delta G$ and $\delta q$. The comparison of these measurements to SIDIS data will allow for very important consistency checks, in particular for a test of factorization in spin-dependent processes.

${ }^{3}$ In the following only the term 'electron' will be used, although most experiments can either be done with electrons or positrons, or with muons in a future high intensity muon collider or neutrino factory.
} 
the addendum to the Technical Design Report 16 for the TESLA project, a $250 \times 250 \mathrm{GeV}$ super-conducting linear $e^{+} e^{-}$collider.

In the case of TESLA-N [17] it is foreseen to inject separately produced (polarized) electron bunches into the positron arm of the TESLA machine and to extract them at one of several possible locations, thereby realizing a beam energy range between 30 and $250 \mathrm{GeV}$. The (polarized) electrons would be directed towards a separate experimental hall. Here a new experiment would combine a (polarized) solid-state target with a new multi-stage spectrometer required due to the large energy range of the produced particles.

In the case of ELFE@DESY [18], separate electron bunches would be accelerated in the first part of TESLA to about $30 \mathrm{GeV}$, transported back and subsequently injected into the HERA- $e$ ring. The latter would serve as stretcher to increase the low TESLA duty cycle of $0.5 \%$ by a substantial factor, whereby keeping a precise energy definition of the beam. In the experiment a (polarized) solidstate target would be combined with a new highresolution spectrometer that will allow for a clear separation of the nucleon ground state from isobars. Its projected performance is illustrated for the exclusive channel $e p \rightarrow e K^{+} Y^{0}$ in Fig. 11 19], assuming that electron and kaon were measured in the spectrometer while the missing mass of the hyperon had to be calculated.

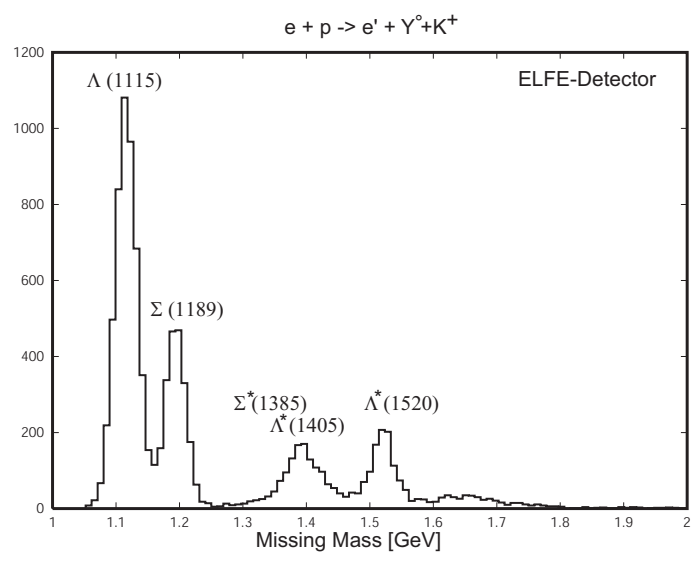

Figure 1. Illustration of the missing mass resolution of a ELFE@DESY-type spectrometer [18,19].
Both concepts may be profitably combined: The technical solution suggested for the beam part of TESLA-N in conjunction with a (polarized) solid-state target and a high-resolution ELFE@DESY-type spectrometer would be ideal to pursue the complex physics programme outlined above. This option could also be realized sharing a lower-energy, 30-50 GeV TESLA-like accelerator that is presently discussed to feed the X-Ray Free Electron Laser facility [20].

In the case that HERA running should be extended beyond 2006, a certain part of this physics programme could already be realized with an upgraded HERMES apparatus.

The envisioned luminosities of both projects, TESLA-N and ELFE@DESY, are depicted in Fig. 2 compared to existing and other possible future facilities. The planned TESLA duty cycle of $0.5 \%$ is the only reason for the lower luminosity limit of TESLA-N in comparison to ELFE@DESY. In any case a luminosity limit of a few times $10^{35} \mathrm{~cm}^{-2} \mathrm{~s}^{-1}$ is imposed by the target cryogenic system when using a polarized solid-state target. This determines also the maximum possible luminosity for a polarized combined TESLA-N/ELFE@DESY experiment once a duty cycle of a few $\%$ is available.

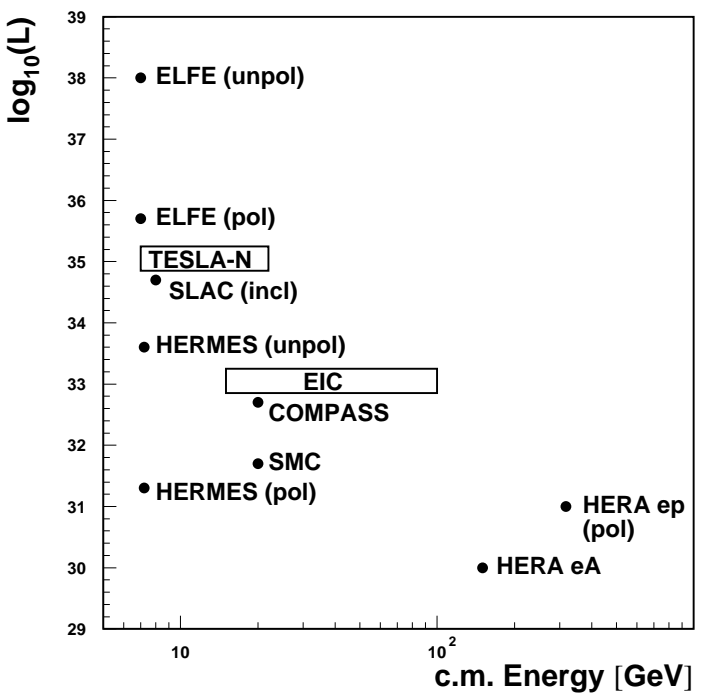

Figure 2. Luminosity versus c.m. energy for existing and (possible) future facilities. 
Note that the effective luminosity of any experiment using a polarized solid-state target, such as the polarized $\mathrm{NH}_{3}$-target of $\mathrm{SMC}$, is lower by a factor of about 25 compared to an electronproton collider (HERA, EIC) or an internalproton-target experiment (HERMES), due to the large dilution factors involved. This dilution effect is not included in Fig. 2.

\section{PROJECTIONS FOR FUTURE MEASUREMENTS}

\subsection{Polarized Gluon Distribution}

In Fig. 3 representative projections are shown for future results on the polarized gluon distribution. Highly precise measurements as planned for RHIC and proposed for TESLA-N are even-

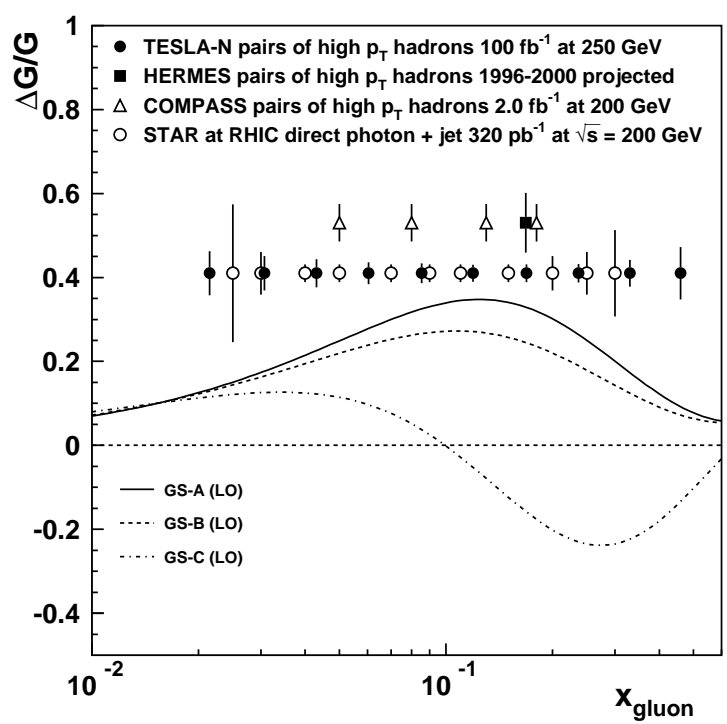

Figure 3. Projected statistical accuracies for the measurement of $\Delta G(x) / G(x)$ at TESLA-N, based on an integrated luminosity of $100 \mathrm{fb}^{-1}$, in comparison to projections from COMPASS [1], HERMES [21] and RHIC [15]. The phenomenological predictions [2] were calculated for $Q^{2}=10 \mathrm{GeV}^{2}$ (taken from Ref. [17]).

tually needed to map out the full $x$-dependence of $\Delta G(x)$ with reasonable precision. The soon expected COMPASS results (including the not shown results from open charm production) will provide very valuable information on $\Delta G(x)$ due to their completely different systematics as compared to RHIC. No projections exist up to now for the accuracy of measurements of $\Delta G\left(x, Q^{2}\right)$.

The RHIC projections shown in Fig. 3 were calculated for a given final state and $\sqrt{s}=200$ $\mathrm{GeV}$, i.e. for $Q^{2}=\mathcal{O}\left(100 \mathrm{GeV}^{2}\right)$. They will exhibit larger error bars, when evolved down to $Q^{2}=10 \mathrm{GeV}^{2}$. At the same time, $\Delta G(x)$ will be determined from other final states and at two different energies. Still, a measurement of $\Delta G(x)$ at RHIC is not without problems in view of the large theoretical uncertainties in direct photon and heavy quark-pair hadroproduction. Hence a statistically highly precise and systematically independent determination of $\Delta G\left(x, Q^{2}\right)$ through an electron-nucleon scattering experiment is clearly needed.

\subsection{Transversity Distributions}

In Fig. 1 the projected statistical accuracy is shown for a measurement of the $\left(x, Q^{2}\right)$ dependence of the $u_{v}$-quark transversity distribution through single-spin azimuthal cross-section

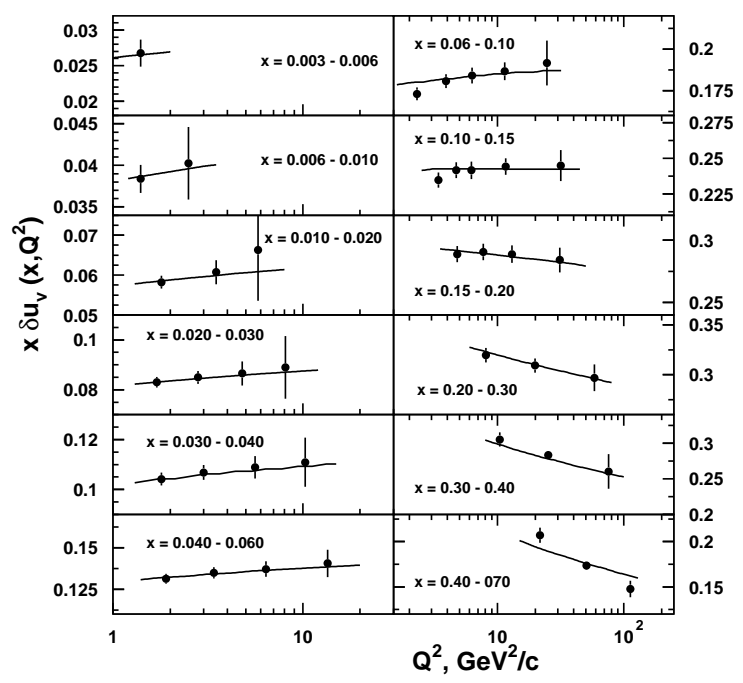

Figure 4. The valence u-quark transversity distribution as a function of $x$ and $Q^{2}$ as it would be measured at TESLA-N, based on an integrated luminosity of $100 \mathrm{fb}^{-1}$. The curves show the $L O$ $Q^{2}$-evolution of the $u_{v}$-quark transversity distribution through a fit to the simulated asymmetries (taken from Ref. [17). 
asymmetries in semi-inclusive charged pion production at TESLA-N [17]. In the simulated analysis it was assumed that transversity and helicity distributions coincide at photon virtualities of the order of $1 \mathrm{GeV}^{2}$ and valence-like $x$-values. Fig. 4 shows that a broad $x$-range $(0.003<x<0.7)$ can be accessed while $Q^{2}$ is in between 1 and $100 \mathrm{GeV}^{2}$, with an impressive statistical accuracy over almost the full range. Because of $u$-quark dominance in pion electro-production a lower accuracy is attained in the reconstruction of the other transversity distributions, $\delta d_{v}, \delta \bar{u}$, and $\delta \bar{d}$.

Measuring the transversity distributions for quarks and anti-quarks of a given flavor would allow for the first time to determine the tensor charge $\delta \Sigma\left(Q^{2}\right)$ of this flavor. This would give access to the hitherto unmeasured chirally-odd operators in QCD which are of great importance to understand the role of chiral symmetry in the structure of the nucleon [23]. A fit to a simulated data sample, as described in Ref. [17], also provides projections for the accuracy of the $u$ - and $d$ quark tensor charges: $0.88 \pm 0.01$ and $-0.32 \pm 0.02$ at a scale of $1 \mathrm{GeV}^{2}$. Note that the absolute values of the tensor charges are determined to a large extent by the input distributions used, although their values are rather close to those predicted by lattice QCD calculations.

\subsection{Generalized Parton Distributions}

The following discussion focuses on DVCS as a tool to derive $J_{q}$ through the determination of GPDs. HERMES plans for 2004-2006 to use an unpolarized target in conjunction with a new recoil detector [24]. As can be seen from the anticipated statistical precision, evaluated in Ref. [25], rather accurate data can be expected on the azimuthal dependence of the asymmetries in beam helicity and beam charge. In addition, for the first time the dependence of these asymmetries on $t$, the third variable on which GPDs depend, may become accessible. Even a first approximate determination of the GPD $H^{u}$ may be tried on the basis of $u$-quark dominance.

Explicit formulae for azimuthal DVCS asymmetries on the twist- 2 level are given e.g. in Ref. [26. In case of measurements with a polarized beam and an unpolarized target the gener- alized parton distribution function $H$ dominates, while the two other involved functions, $\tilde{H}$ and $E$, are suppressed relative to $H$ by kinematic factors.

Determining through Ji's relation [9] the total angular momentum $J_{q}$ carried by quarks of flavor $q$ requires data not only on the helicityconserving GPD $H$, but as well on the helicity-flip GPD $E$. Access to $E$ may be achieved through DVCS measurements with an unpolarized beam and a transversely polarized target. Also, important additional knowledge on $E$ can be expected from exclusive vector meson production.

Running with a polarized internal target is presently still technologically disfavored, because the maximum achievable instantaneous luminosity falls short by about two orders of magnitude compared to unpolarized targets. In the case of HERMES running beyond 2006, a polarized target with a density improvement by about a factor of two may become available.

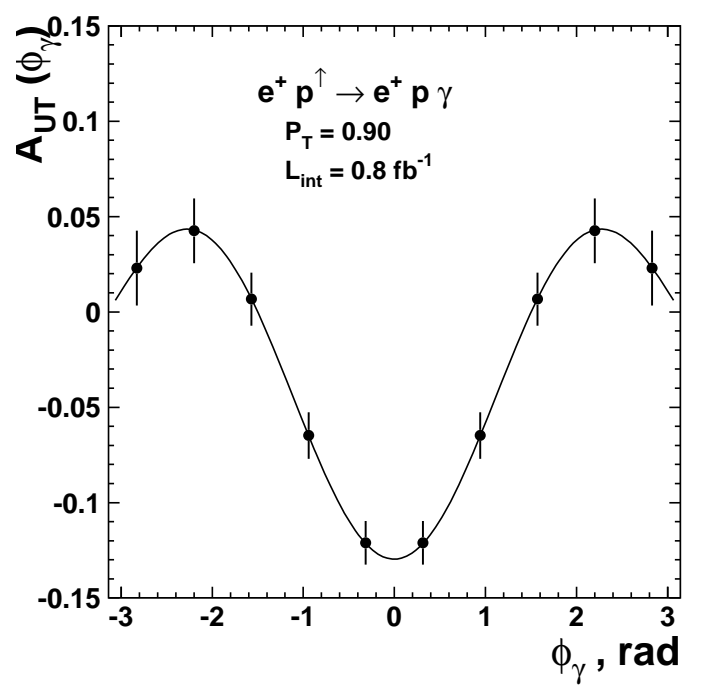

Figure 5. Projected statistical accuracy for the azimuthal dependence of the DVCS asymmetry in a low-luminosity experiment with unpolarized electron beam and transversely polarized target [27], based upon the HERMES acceptance.

From Fig. 5 it can be seen that the statistical precision, achievable after three years of HERMES running with an unpolarized beam and a transversely polarized target, would be sufficient to measure the azimuthal dependence of the 
DVCS asymmetry $A_{U T}$ [27. The curve depicts the behaviour of the chosen GPD parameterization (B), as explained in detail in Ref. [25]. Note that its missing periodicity in $\phi_{\gamma}$ is due to a particular way of integrating over one of the azimuthal angles in Ref. [26]. It remains to be shown that, even using the approximation of $u$-quark dominance, this data set would allow simultaneous access to the GPDs $H$ and $E$. Extrapolating them to $t=0$ and simultaneously calculating the integral over $x$ would make it possible in principle to obtain a first glimpse of the total angular momentum carried by $u$-quarks, $J_{u}$. However, this may not be feasible because of lack of statistics and several theoretical uncertainties that have to be quantified reliably.

A real breakthrough towards a determination of GPDs can be expected only from high-statistics measurements at a future high-luminosity facility, as outlined above. A typical rather conservative assumption on integrated luminosity is $100 \mathrm{fb}^{-1}$ per year of running (cf. Ref. [17]). As explained above, a key experiment would consist in measuring DVCS with an unpolarized beam and a transversely polarized target. In this case a typical observable would be the $\cos (\phi / 2)$ moment of the cross section asymmetry that projects out the set of GPDs as discussed above.

In Fig. 6 the statistical accuracy is illustrated for a measurement of DVCS in a $30 \mathrm{GeV}$ electron beam with a high-resolution spectrometer, combined with a $\mathrm{NH}_{3}$-target ( $80 \%$ polarization, dilution factor $17.6 \%$ ) and applying the same cuts as used in the analysis of Ref. 111. The error bars show the projected statistical accuracy achievable in 1 year of running for the $x_{B}$-dependence of the moment $A_{U T}^{\cos (\phi / 2)}$ introduced above. The curve again represents the behaviour of the GPD parameterization (B) 257. The statistical errors clearly show that an acceptable precision can be reached and that even the measurement of 2dimensional dependences (e.g. on $x_{B}$ and $t$ ) are within reach. It remains to be shown by a careful analysis of simulated data that the ultimate goal, the above envisioned 'complete' determination of the angular momentum structure of the

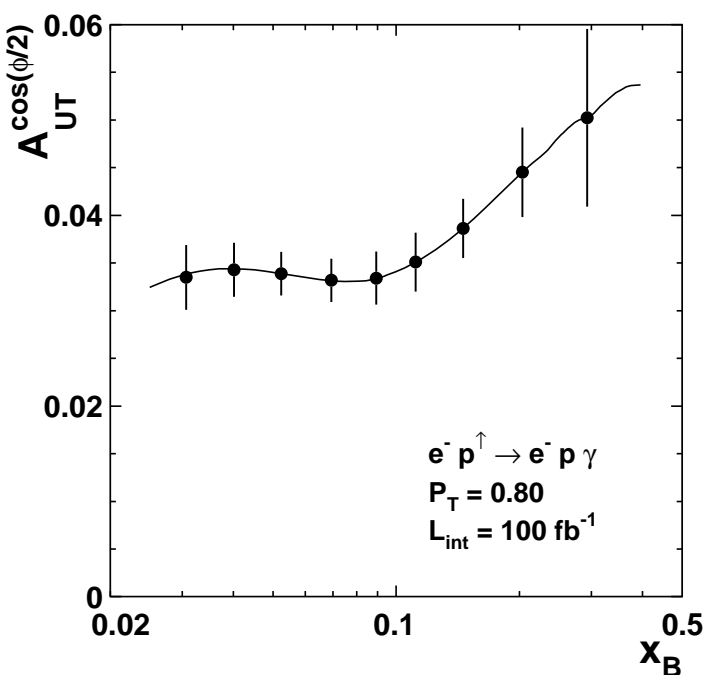

Figure 6. Projected statistical accuracy for the $x_{B}$-dependence of the $\cos (\phi / 2)$ moment in a highluminosity experiment with unpolarized electron beam and transversely polarized target [27], using a conical acceptance between 40 and 220 mrad.

nucleon, can be experimentally achieved and that the most complex variety of theoretical uncertainties can be brought under control to make this high-statistics result meaningful.

\section{SUMMARY AND CONCLUSION}

In the context of the proposed TESLA/FEL project, a new high-luminosity high-resolution electron-nucleon facility may be accommodated. This would pave the way for a great variety of measurements, ranging from inclusive scattering through semi-inclusive final states to hard exclusive processes. On the basis of the anticipated statistical accuracies, shown for various key experiments, it appears justified to expect that many questions on the structure of hadronic matter, especially on its momentum and spin structure, can be successfully answered.

It goes without saying that there will be lots of further extremely interesting physics results beyond those discussed above. Among the published HERMES results, after 5 years of running, only $10-20 \%$ had been anticipated in the proposal. 


\section{ACKNOWLEGDEMENTS}

I am deeply indebted to V.A. Korotkov for calculating new DVCS projections, to A. Miller for a very careful reading of the manuscript and to D. von Harrach for an update of Fig. 1. Many thanks to them and to S. Bass, M. Diehl, P. Hoyer, D. Müller, D. Ryckbosch and G. van der Steenhoven for very valuable comments. The help of R. Kaiser in the preparation of Fig. 2 is acknowledged.

\section{REFERENCES}

1. J. Nassalski, Acta Phys. Pol. B29 (1998) 1315

2. M. Beckmann, Proc. of the 9th Int. Workshop on Deep Inelastic Scattering (DIS2001), World Scientific, to appear.

3. J.P. Ralston, D.B. Soper, Nucl. Phys. B152 (1979) 109

4. X. Artru, M. Mekhfi, Z. Phys. C45 (1990) 669

5. R.L. Jaffe, X. Ji, Phys. Rev. Lett. 71 (1993) 2547

6. V.A. Korotkov, W.-D. Nowak, K. Oganessyan, Europ. J. Phys. C18 (2001) 639

7. D. Müller et al., Fortschr. Phys. 42 (1994) 101

8. A.V. Radyushkin, Phys. Lett. B380 (1996) 417; Phys. Rev. D56 (1997) 5524

9. X. Ji, Phys. Rev. Lett. 78 (1997) 610; Phys. Rev. D55 (1997) 7114

10. J. Blümlein et al., Nucl. Phys. B560 (1999) 283; Nucl. Phys. B581 (2000) 449.

11. HERMES Coll., A. Airapetian et al., Phys. Rev. Lett. 87 (2001) 182001

12. CLAS Coll., S. Stepanyan et al., Phys. Rev. Lett. 87 (2001) 182002

13. H1 Coll., C. Adloff et al., Phys. Lett. B 517 (2001) 47

14. L.L. Frankfurt, A. Freund, M. Strikman, Phys. Rev. D58 (1998) 114001; Phys. Rev. D59 (1999) 119901E (erratum)

A. Donnachie, H.G. Dosch, Phys. Lett. B502 (2001) 74

15. L. Bland, Proc. of the Workshop 'Physics with a High Luminosity Polarized Electron Ion Collider' (EPIC99), World Scientific, ed.s L. Bland et al., p.255 (2000); hep-ex/9907058

16. TESLA Technical Design Report, DESY
2001-011, Part I, Executive Summary, ed.s F. Richard et al.

17. ibid., Part VI, Appendices, TESLA-N: Electron Scattering with Polarized Targets at TESLA, ed.s R. Kaiser, W.-D. Nowak; also in: M. Anselmino et al., DESY 00-160, hep$\mathrm{ph} / 0011299$

18. ibid., Part VI, Appendices, ELFE: The Electron Laboratory for Europe, ed.s E. DeSanctis, J.-M. Laget, K. Rith; also in: M. Anselmino et al., ELFE: Physics Motivations, NuPECC Report, Sept. 2001

19. D. von Harrach, priv. comm.

20. ibid., Part V, The X-Ray Free Electron Laser, ed.s G. Materlik, Th. Tschentner

21. E. Aschenauer, priv. comm.

22. T. Gehrmann, W.J. Stirling, Phys. Rev. D53 (1996) 6100

23. R.L. Jaffe, MIT-CTP-2685, hep-ph/9710465

24. HERMES Coll., A Large Acceptance Recoil Detector for HERMES, DESY PRC 01-01, April 2001.

25. V.A. Korotkov, W.-D. Nowak, DESY 01-109, hep-ph/0108077, Europ. J. Phys. C., in press

26. A.V. Belitsky et al., Nucl.Phys. B593 (2001) 289

27. V.A. Korotkov, priv. comm. 